\title{
Extended throat venturi based flow meter for optimization of oil production process
}

\author{
Muhammad Akram Karimi, Student Member, IEEE, Muhammad Arsalan, and Atif Shamim, Senior Member, IEEE
}

Abstract - Water is mixed with the crude oil to form complex multiphase fluid during oil extraction process. Some of the traditional methods to measure the relative amount of water mixed with the crude oil (known as water-cut or WC) are intrusive, which are prone to wear and tear. Few other methods, used for WC sensing, are either unable to cover full range ( 0 $100 \%$ WC) or require good mixing of the production fluid to be sensed accurately. This paper presents a unique and robust design of a dual spiral microwave resonator, which has been integrated on the extended throat of a venturi. Venturi measures the flow rate of the overall fluid while the microwave

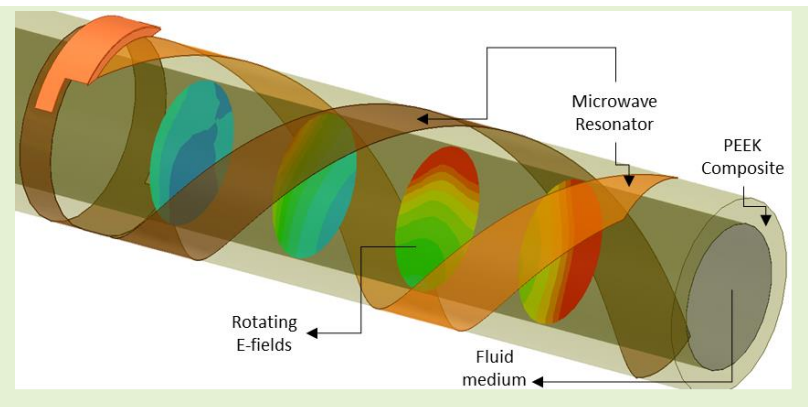
resonator measures the relative fraction of water in oil. Unlike existing meters, the presented design is fully nonintrusive, covers full WC range and does not require any mixing of production fluid for accurate measurement. The meter has been designed to withstand harsh field conditions of 1000 psi pressure and $125^{\circ} \mathrm{C}$ temperature and its performance has been validated in a commercial industrial flow loop with variable salinity conditions. "Phase inversion" phenomenon where the mixture changes from "oil continuous" to "water continuous" or vice versa, has been characterized thoroughly under varying test conditions. Microwave sensor's resonance frequency $\left(f_{0}\right)$ and quality $(Q)$ factor are used to measure WC in oil continuous and water continuous conditions respectively. The meter has been tested over wide range of flow rates and full range $(0-100 \%)$ of WC. Liquid flow rate accuracy of $\pm 3 \%$ and water cut accuracy of $\pm 2 \%$ has been obtained from the industrial flow loop measurements.

Index Terms - Flow meter, Industrial flow loop, Microwave resonator, Venturi, Water-cut sensor

\section{INTRODUCTION}

$\mathrm{W}$ ATER is the largest by-product of oil production process. On average, 3 barrels of water are produced for every barrel of oil [1]. Oil industry spends more than 40 billion USD every year in order to manage the unwanted water [2]. Sudden rise in water level is an indication of many problems related to an oil well. Some of those problems can be casing leakage, water coning or fractures/faults between water injection and oil producing wells. It is therefore very important to keep track of exact amount of water production from every well so that preventive measures can be taken to minimize the impact, which can be very costly if not tackled immediately.

\section{A. State of the art water-cut meters}

There are many existing techniques to measure the waterfraction in oil by exploiting the difference in density [3], $\mathrm{THz}$ wave absorption [4], IR waves absorption [5], dielectric constant [6] [7], dielectric loss [8] or conductivity [9] characteristics of oil and water. All of these techniques have

\footnotetext{
Manuscript received on November 23, 2020. (Corresponding author: Muhammad Akram Karimi)

M. A. Karimi and A. Shamim are with Integrated Microwave Packaging Antennas and Circuits Technology, Computer, Electrical, and Mathematical Sciences and Engineering Division, King Abdullah University of Science and Technology, Thuwal 23955, Saudi Arabia (email: muhammadakram.karimi@kaust.edu.sa). M. Arsalan is with the Production Technology Team, EXPEC ARC, Saudi Aramco 31311, Saudi Arabia (e-mail: muhammad.arsalan@aramco.com).
}

some drawbacks and active research is going on to overcome these challenges. For example, Coriolis meter measures the WC based on density difference of oil and water but the meter becomes very bulky for pipe sizes larger than 3 ". Recent studies have shown the use of off-the-shelf $\mathrm{THz}$ cameras to differentiate water and oil mediums owing to difference in their absorption characteristics [4]. However, this technique is limited by slow speed and low resolution of currently available THz imaging systems [11]. Moreover, limited signal penetration (esp. at high $\mathrm{THz}$ frequencies) and wave refraction from fluid particles are also amongst the challenges faced by $\mathrm{THz}$ based WC measurements.
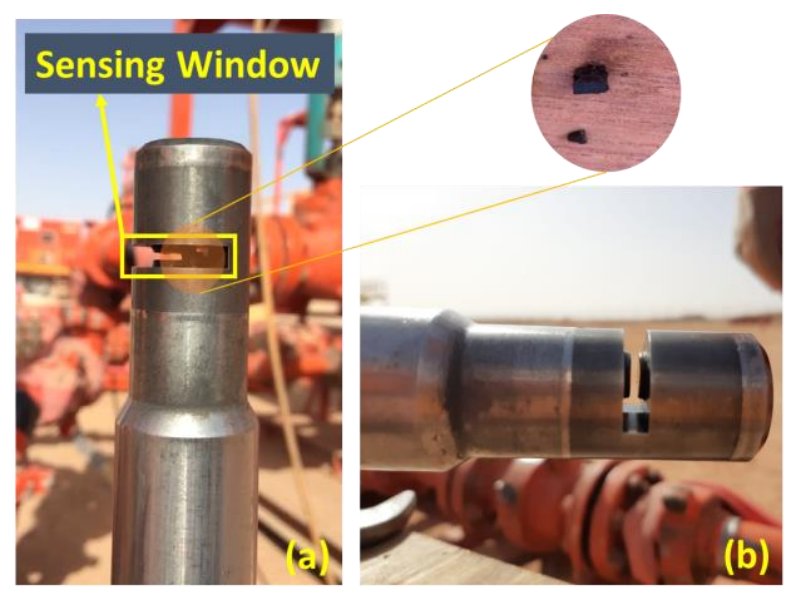

Fig. 1. (a) Sensing window stuck with black particle (b) sensing window in cleaned form 
Non-intrusive WC measurement techniques ensure longterm serviceability which is possible to achieve with all the WC measurement principles except infrared (IR) based measurements. IR waves cannot propagate long distance in the fluid, which is why such meters have a small sensing window usually in the middle of the pipeline, which can be clogged with solid sand particles, resulting in highly inaccurate measurements and expensive downtime of field operation.

Authors have personally experienced similar IR WC meter on the oil field, which was clogged with solid black particle resulting into down time of many hours and wrong reading for many days. Fig. 1(a) shows an IR WC meter with foreign object stuck into its sensing window while Fig. 1(b) shows the scenario when the particle was removed from the instrument.

All other types of WC measurements, based on dielectric properties (dielectric constant, dielectric loss, conductivity) of water and oil are heavily dependent upon a phenomenon called "phase inversion". At low WC value (<40-60\%), 2phase oil/water liquid exhibits "oil continuous" properties in which oil forms the continuous film with water particles suspended inside it. While, at high WC values (>40-60\%), water forms the continuous film and the 2-phase fluid is regarded as "water continuous". The phenomenon of moving from oil-continuous to water-continuous or vice versa is known as "phase inversion". 2-phase fluid is non-conductive in oil continuous phase and acts as conductive medium in water continuous phase, which is why the knowledge of continuous phase is very important for accurate WC measurements using dielectric techniques. Capacitance only and conductance only WC meters are limited to operate in oil continuous and water continuous region respectively. This is the reason that most of modern WC meters are combining capacitance and conductance measurements in an effort to cover full WC range. However, accurate detection of continuous phase remains a challenge.
This paper presents a microwave method of detecting the difference in dielectric properties (dielectric constant and dielectric loss) of oil and water. Microwave is a proven technology, which has traditionally been used to measure material's dielectric properties for various applications [12] $[13][14][15][16][17]$. Unlike capacitance and conductance based WC measurements, microwaves allow the use of multifrequency measurements which opens up possibility of detecting additional fluid characteristics such as water salinity and gas volume fraction to name a few. In addition to that, the sensor presented in this paper also shows a technique to detect the continuous phase, which helps in applying correct algorithm to measure the WC value.

Phase inversion phenomenon is affected by many parameters such as flow rate and salinity of the produced water, etc. This paper also shows the characterization of the microwave sensor over a wide range of flow rates and variable salinity levels in order to obtain relationships of various parameters with the phase inversion and to get the calibration factors for variable salinity levels. Moreover, the WC sensor design presented in this paper is completely non-intrusive which ensures long operational life by avoiding meter's downtime as well as the effects of corrosion.

\section{B. State of the art 2-phase flow meters}

In addition to measuring water fraction in oil, their flow rates are also important parameters to be measured. For that purpose, several differential pressure based flow measurement devices such as a venturi or an orifice plate are used.

Some researchers have combined a flow measurement device with WC measurements. For example, Chao et al. has combined cone flow meter with a pair of conductance rings used as a WC meter [18]. However, this integration is intrusive to the fluid flow and conductance rings can measure WC only in the water-continuous region. Mayela et al. has used coriolis measurements as a 2-phase flow meter but it becomes bulky for pipe sizes greater than 3" [19].

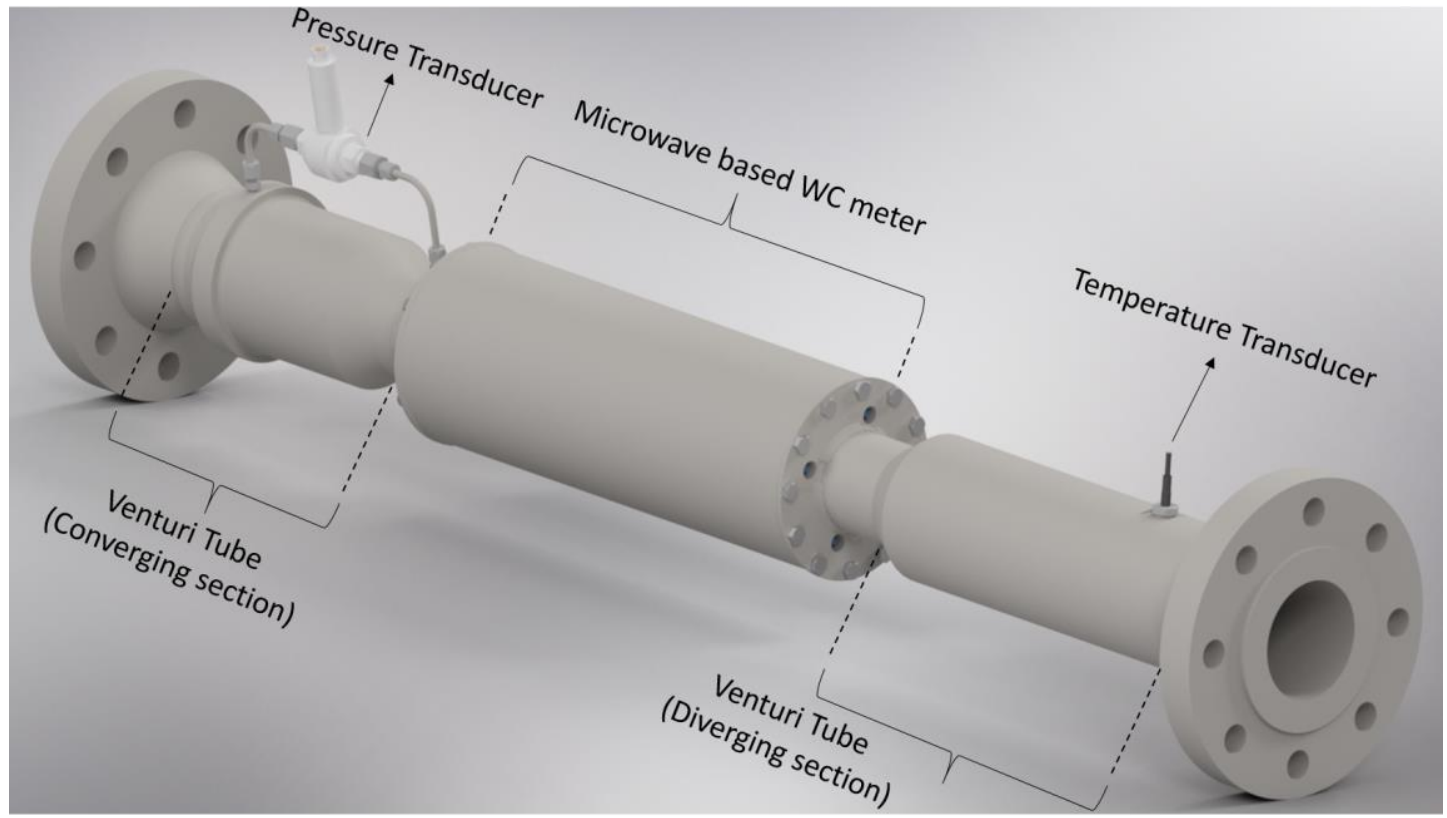

Fig. 2. Proposed 2-phase flow meter with extended throat venturi and embedded microwave based WC measurements 
This paper shows a unique integration of microwave based WC measurement method with the venturi flow meter. The novel integration ensures non-intrusive and full range WC and flow rate measurement of oil and water. All of these features have been achieved while maintaining the robustness of the device, which ensures its serviceability in the harsh field conditions.

\section{SENSOR DESIGN}

Traditional venturi meters are extensively used in the oil fields for measuring the flow rate of single phase or multiphase fluids [10], but they do not have the capability to measure the fraction of water in oil. Unlike a traditional venturi, we have extended its throat and used it as a placeholder for microwave based WC meter. By doing this, we were able to measure flow rate as well as the WC value of 2-phase fluid using a single device. The venturi presented in this paper has relatively long throat in order to accommodate the WC sensor laterally, which is why the design is called "extended throat venturi". Venturi with a long throat is also useful to measure complex flows, encountered in oil and gas industry. For example, $\mathrm{Xu}$ et al. has studied extended throat venturi to measure wet gas flow, without using any phase fraction technique [20]. The unique design presented in this paper opens up the possibility to measure wet gas flows more accurately by embedding microwave based water and/or gas fraction meter on the extended throat of the venturi.

\section{A. Extended throat venturi for flow measurements}

The cross section view of extended throat venturi based 2phase flow meter is shown in Fig. 3. The flow meter has inlet diameter of $4 "$, which is reduced to $1.6^{\prime \prime}$ in order to create pressure differential in proportion to the flow rate of the fluid. The converging and diverging sections of venturi tube have been designed as per ISO 5167-4 standards, which follows the standard venturi relationship given by equation 1 .

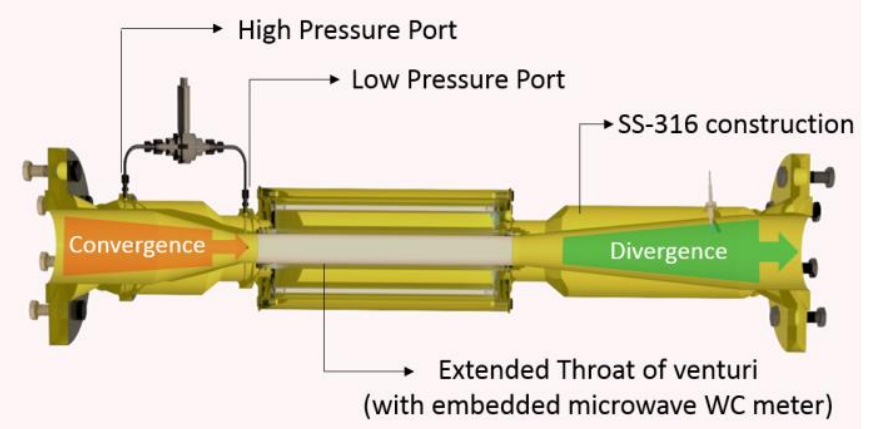

Fig. 3. Cross section view of extended venturi based 2-phase flow meter

Where,

$$
q_{v}=\frac{C}{\sqrt{1-\beta^{4}}} \varepsilon \frac{\pi}{4} d^{2} \sqrt{\frac{2 \Delta p}{\rho_{1}}}
$$

- Diameter ratio: $\beta=\frac{d}{D}=\frac{1.6 \mathrm{in}}{4.026 \mathrm{in}} \cong 0.4$

- $\Delta p$ is the differential pressure measured by the pressure transducer

- $\rho_{1}$ is the fluid density at the temperature and pressure in entrance cylinder

- Expansibility factor: $\varepsilon \sim 1$

In the presence of 2-component (oil \& water), fluid density $\left(\rho_{1}\right)$ is a function of water-fraction or WC which is measured by microwave WC meter embedded in extended throat of venturi whose design in explained in the next section.

\section{B. Microwave sensor for WC measurements}

The proposed meter utilizes "Dual Mutually Orthogonal Resonators" operating in low microwave frequency band in the range of $135-220 \mathrm{MHz}$ to measure the WC value of 2phase fluid. The sensor is comprised of two spiral resonators, which are oriented orthogonal to each other as can be seen from Fig. 4.

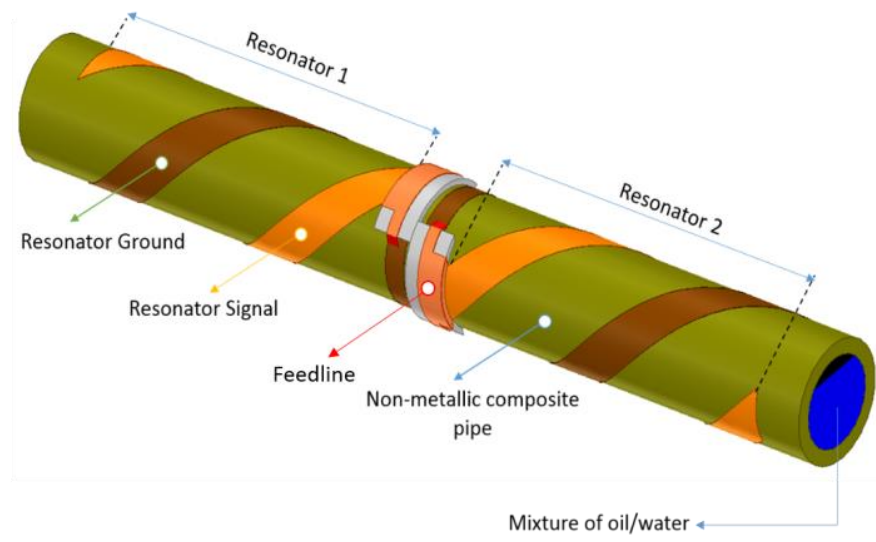

Fig. 4. "Dual mutually orthogonal resonator" based WC sensor design operating in low microwave frequency band

Each microwave resonator has a feedline with two ports. Microwave signal is injected from one port, which experiences maximum reflection at a frequency, called resonance frequency $\left(\mathrm{f}_{0}\right)$. At $\mathrm{f}_{0}$, very little amount of microwave signal is transmitted from one port to the other which is why the transmission coefficient $\left(\mathrm{S}_{21}\right)$ of the resonator shows band-stop response, as shown in Fig. 5. As water has higher dielectric constant $(\sim 80)$ than crude oil $(\sim 2.2)$, it slows down the microwaves and $\mathrm{f}_{0}$ value decreases with increasing amount of water in oil or WC value. It can also be noted from Fig. 5 that resonance curve becomes broader (larger bandwidth) at high WC values. Broader resonance is an indication of higher dielectric loss, which is calculated using a parameter called Qfactor of the resonator. Q-factor of the microwave resonator decreases with increasing dielectric loss or WC value of 2phase mixture.

$\mathrm{f}_{0}$ value is used to determine $\mathrm{WC}$ in oil continuous region and Q-factor is used to determine WC in water continuous region, as will be explained further in "testing" section.

- $q_{\bar{\Sigma}}$ : volumetric flow rate of fluid

- Discharge coefficient: $C=0.995$ 


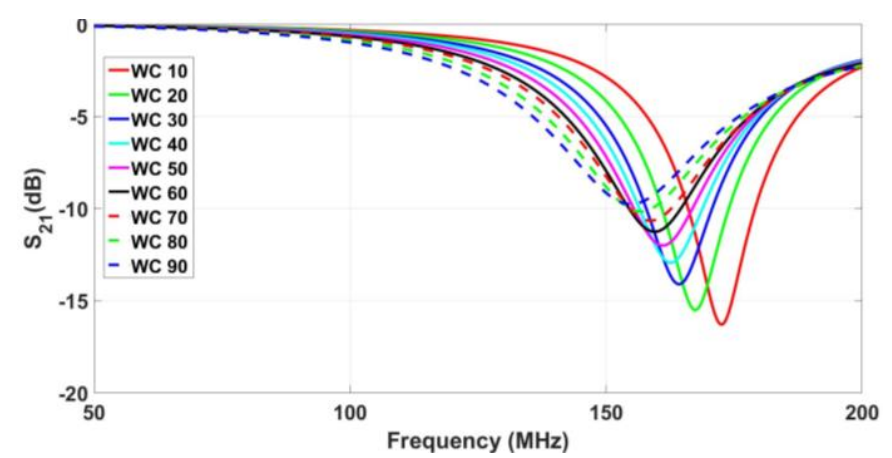

Fig. 5. Simulated response of the sensor over full WC range in the presence salty water with 200,000 ppm concentration

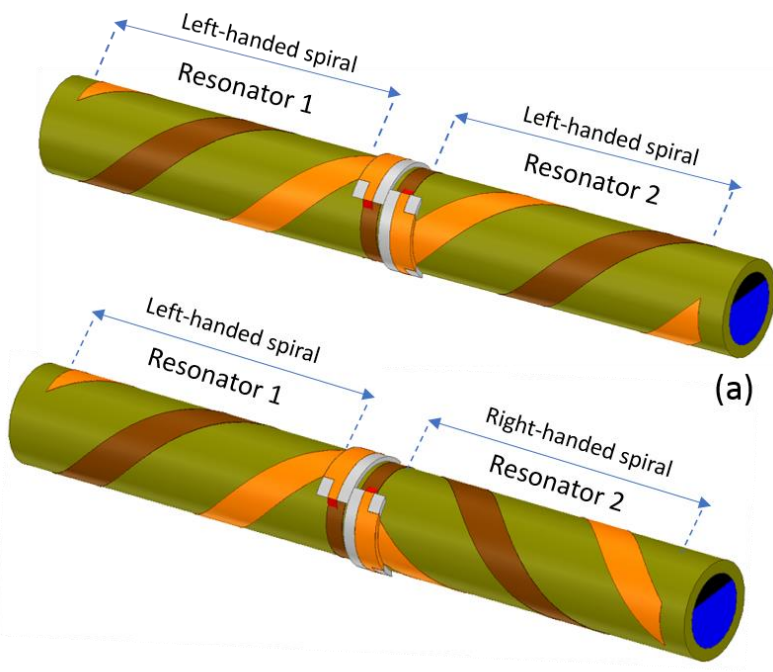

(b)

Fig. 6 (a) Conventional dual resonator design with both resonators having left-handedness (b) Improved sensor design with one resonator with left-handedness and other with right-handedness
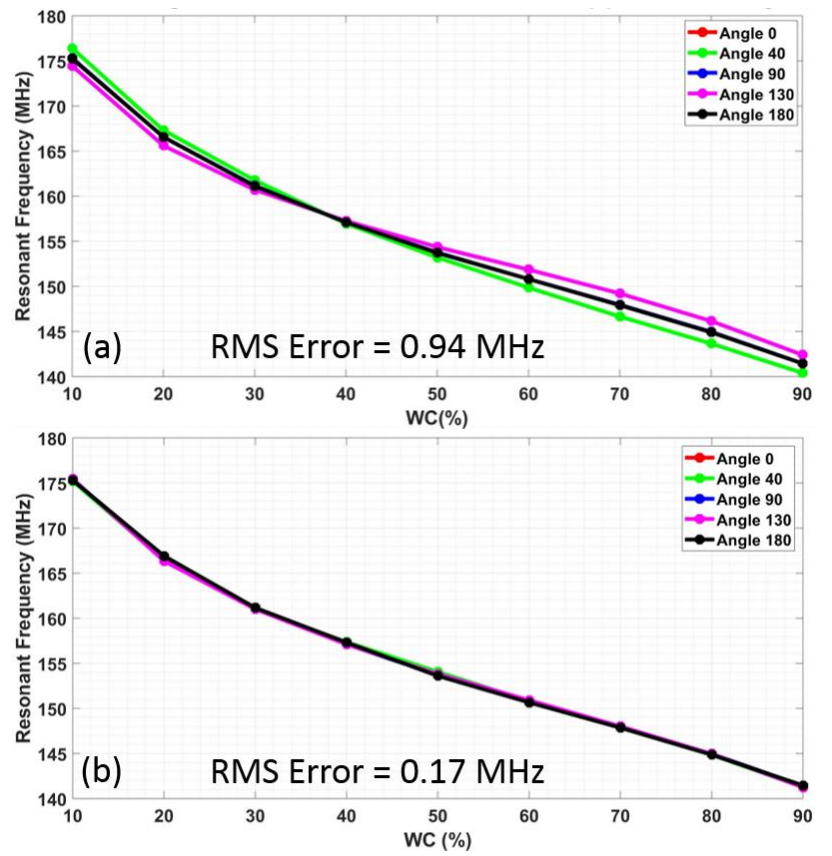

Fig. 7. Average of resonant frequencies of two mutually orthogonal resonators plotted against WC for various orientations of oil and water. Water has conductivity of $5 \mathrm{~S} / \mathrm{m}$, which corresponds to salinity concentration of $\sim 30,000$ ppm. (a) Design 1 with both spirals having left-handedness (b) Design 2 with one left-handed and other right handed spiral resonator

The advantage of using dual orthogonal resonators is that the arithmetic average of resonance frequecnies $\left(f_{0-a v g}\right)$ of two resonators, as given by equation (2), remains the same irrespective of geometric distribution of oil and water inside the pipe [7]. That is why; the presented sensor configuration gives orientation insensitive WC reading without any need to mix oil and water together.

$$
\mathrm{f}_{\mathrm{o}-\text { avg }}=(1 / 2)\left[\mathrm{f}_{\mathrm{o} 1}+\mathrm{f}_{\mathrm{o} 2}\right]
$$

Where,

$\mathrm{f}_{\text {o-avg }}=$ Averaged resonance frequency, used for WC calculation

$f_{01}=$ Resonance frequency of resonator \# 1

$\mathrm{f}_{\mathrm{o} 2}=$ Resonance frequency of resonator \# 2

Orientation insensitivity is a key feature of this sensor configuration, which depends on the handedness of the helical resonators used in the sensor. Both the resonators used in previously reported sensor design (shown in Fig. 6(a)) are left handed. However, HFSS simulations show that if two resonators have opposite handedness (one left-handed and other right-handed spiral as shown in Fig. 6(b)), orientation insensitivity can be improved by a factor of 5.4.

Comparison of frequency responses of two designs, which were simulated for different orientations of oil and water, is shown in Fig. 7. Conventional configuration with left-handed spiral resonators (Fig. 6(a)) does not give very good orientation insensitivity as evident from Fig. 7 (a). This is because two spiral resonators with same handedness are effectively single resonator with two turns which does not help much to distribute $\mathrm{E}$ fields in the sensing medium uniformly.

On the other hand, the sensor design with opposite handed spirals (Fig. 6(b)) can measure the WC irrespective of oil and water orientation inside the pipe as evident from Fig. 7(b). This is because E fields of opposite handed resonators penetrate in sensing medium from two different directions which help distribute $\mathrm{E}$ fields more uniformly in pipe cross section and hence achieving better orientation insensitivity.

Since the oil and gas fields produce water with high amounts of salts mixed with it. In this paper, we will test the improved version of the sensor in saline water conditions. It is a common misconception that microwave resonance phenomenon may fail at high salinity levels due to increased dielectric loss of the fluid medium [21]. In order to verify that the WC sensor works fine even at high salinity levels, we have simulated the sensor in HFSS at extremely high salinity level of $200,000 \mathrm{ppm}$. Simulated transmission parameter $\left(\mathbf{S}_{21}\right)$ of microwave resonator (shown in Fig. 5) indicates that the resonance is quite prominent over wide range of water-cut (WC) and the inverse relationship of resonance frequency $\left(f_{0}\right)$ and WC value holds true. It can also be noted that Q-factor decreases as the WC value is increased.

\section{Mechanical Strength Evaluation of the Meter}

In order to ensure the mechanical integrity of the meter, finite element analysis (FEA) of all of its parts has been performed. The presented meter is rated for 1000 psi pressure, and we have applied a pressure of 2000 psi in order to keep a safety margin of $2 \mathrm{x}$ and observed the stress on various parts of 
the meter. The stress is carefully observed at the critical points such as weldments and the part edges, as shown in Fig. 8. Important thing to consider is that the maximum stress observed at all parts of the meter, must be less than the yield strength of the material. It can be seen from Fig. 8 (a), (c) and (d) that the maximum stress observed on the metallic SS-316 parts is around $17 \mathrm{k}$ psi which is much less compared to the yield strength of the 316 Stainless Steel (UNS31600 per ASTM A276), which is 30k psi. Similarly, maximum stress observed on PEEK tube (shown in Fig. 8 (b)) is around 12.5k psi, which is much less compared to its yield strength of $16.5 \mathrm{k}$ psi. FEA analysis shows that meter is safe to operate at 1000 psi pressure with a safety margin of $2 \mathrm{x}$.

\section{FABRICATION AND TESTING}

\section{A. Fabrication}

After validating the simulated RF performance and mechanical strength of the design, two prototypes of the meter have been built, as shown in Fig. 9. The two copies are exactly the same expect that one of the meters make use of Kalrez Orings, which is much more $\mathrm{H}_{2} \mathrm{~S}$ resistant than typically used Orings made of Viton. Kalrez based prototype is recommended to be used in $\mathrm{H}_{2} \mathrm{~S}$ environment if the meter has to be tested in slightly sour oil wells.

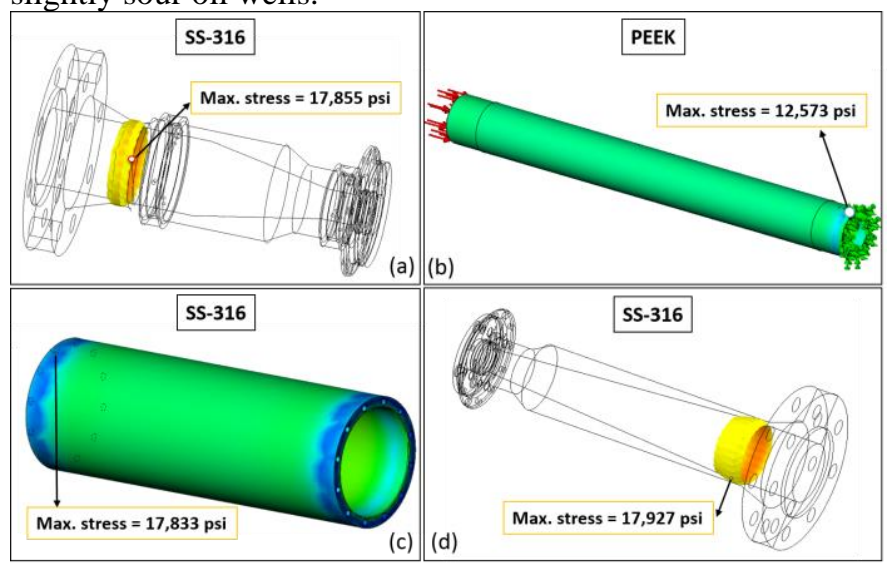

Fig. 8. Stress analysis of various mechanical parts of the meters under 2000 psi pressure (a) Entrance converging cylinder (b) Extended throat of venturi (c) Protective pipe around PEEK tube (d) Exit diverging cylinder

With the help of silver paste, microwave WC meter has been screen-printed on the external surface of the PEEK tube as shown in Fig. 9 (a). In order to check the temperature rating of the WC sensor, PEEK tube along with the printed sensor, have been exposed to temperature as high as $125^{\circ} \mathrm{C}$ for 24 hours. The purpose of this step is to ensure that the metal does not peel or crack off the surface due to mismatch of thermal expansion coefficient of PEEK and silver paste material.

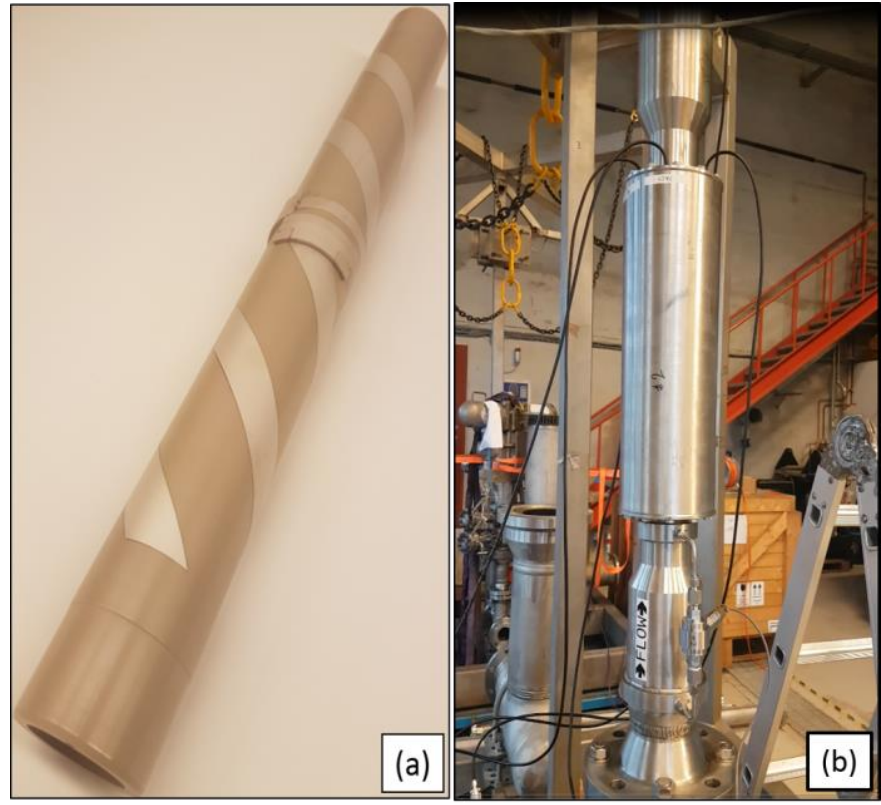

Fig. 9. Fabricated industrial grade prototype of 2-phase flow meter (a) Printed microwave sensor for WC measurements (b) Complete meter assembly being tested in Industrial flow loop of NORCE, Norway

\section{B. Industrial flow loop testing}

Industrial flow loop is a closed loop setup, which circulates oil, water and gases at adjustable flow rates to test the performance of the meter before its deployment in an oil or gas field. We have used NORCE (previously CMR) multiphase flow loop in order to test 2-phase flow performance of our meter using the test setup shown in Fig. 10 [22].

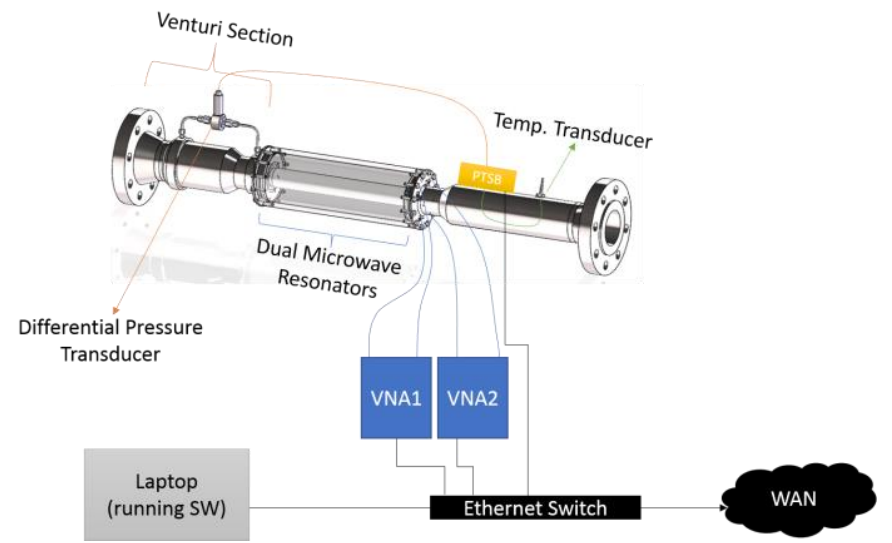

Fig. 10. Block diagram of test setup of 2-phase flow meter. 'VNA' stands for 'vector network analyzer' used to analyze transmission coefficient (S21) of microwave resonator while 'PTSB' stands for 'pressure temperature sensor box', which translates pressure and temperature transducer readings into appropriate format

As explained earlier that one of the key challenges in dielectric multiphase flow sensing is the "phase inversion" phenomenon, which can happen at any value of WC between $30 \%$ to $70 \%$. "Phase inversion" point is hard to predict because it depends on various operating parameters such as salinity level, temperature, pressure, flow rate, pipe roughness and gas fraction (if any). It is worth mentioning that 'tap water' cannot really emulate the 'phase inversion' especially in water-continuous region. That is why; 'saline water' with varying salinity levels has been used during industrial flow 
testing to accurately emulate the field conditions. The physical properties of Gasoil -11 LSHO 10ppm from Exxon Mobil are also very close to 'crude oil', which has been used during 2phase flow testing.

At a particular test condition, WC value has been increased from $0 \%$ to $100 \%$ (known as 'ramp-up') and then decreased from $100 \%$ to $0 \%$ (known as 'ramp-down') in order to verify the repeatability of measurements as well as to observe the 'hysteresis' phenomena, which will be explained in upcoming sections.

\section{1) Effect of flow rate on phase inversion}

We have investigated the effect of flow rate on phase inversion phenomenon, in our first set of measurements. Sensor's resonance frequency $\left(f_{0}\right)$ has been recorded by ramping up the (shown in red curve of Fig. 11) and ramping down (shown in green curve of Fig. 11) the WC value at low flow rate of $20 \mathrm{~m}^{3} / \mathrm{hr}(3000 \mathrm{bpd})$. In Fig. 11, we can observe an abrupt change in f0 at WC $60 \%$ in the red curve and steep change in $\mathrm{f}_{0}$ at WC $25 \%$ in the green curve. The sharp change in $\mathrm{f}_{0}$ indicates that oil continuous medium has been converted into water-continuous medium and vice versa.

This experiment has been repeated at a higher flow rate of $50 \mathrm{~m}^{3} / \mathrm{hr}(7,500 \mathrm{bpd})$ whose response is shown in Fig. 12. It can be observed from Fig. 11 and Fig. 12 that irrespective of the flow rate, $\mathrm{f}_{0}$ value of the sensor in oil continuous and water continuous regions are non-overlapping which results into a 'distinguishing gap' and helps in identifying the continuous region based on sensor's $f_{0}$ value. Distinguishing gap is however small at low flow rate (Fig. 11) as compared to what is observed at higher flow rate (Fig. 12).

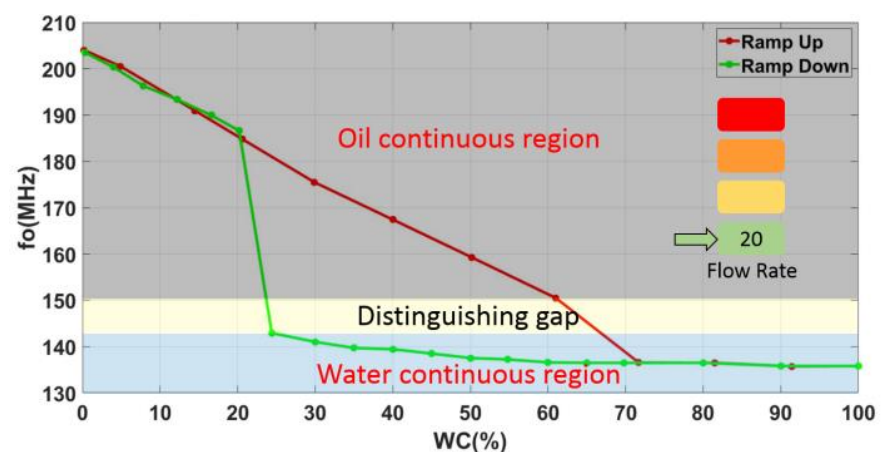

Fig. 11. Hysteresis effect of WC sensor at low flow rate (20 $\mathrm{m} 3 / \mathrm{hr})$

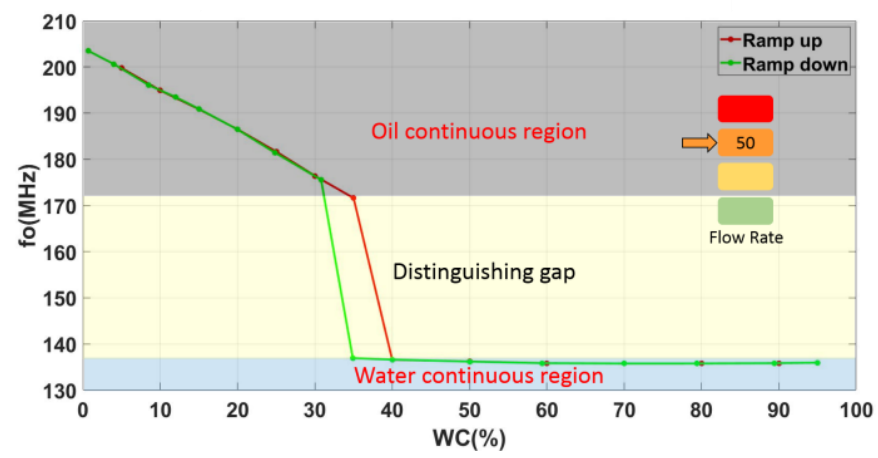

Fig. 12. Hysteresis effect of WC sensor at medium flow rate $(50 \mathrm{~m} 3 / \mathrm{hr})$
It is therefore much easier to identify oil and water continuous region at higher flow rates.

\section{2) Water-cut detection in oil-continuous region}

Once the fluid is detected as "oil continuous", $\mathrm{f}_{0}$ value of the sensor can be related with the $\mathrm{WC}$ value as $\mathrm{f}_{0}$ has a linear relationship with WC as evident from Fig. 11 and Fig. 12.

\section{3) Water-cut detection in water-continuous region}

Resonant frequency $\left(\mathrm{f}_{0}\right)$ of the sensor does not change significantly in water continuous region due to "water blanketing phenomenon" as is evident form Fig. 11 and Fig. 12. This is because, in water continuous region; high electric permittivity medium (water) envelops the low permittivity medium (oil). It is well known that electric fields tend to adopt high permittivity (water) path, and will not experience low permittivity (oil) phase because it has been shielded by water. A dummy figure to explain the water-blanketing phenomenon is shown in Fig. 13. Imaginative E-fields have been drawn in the figure showing their tendency towards high permittivity medium. Changing water fraction (or WC value) does not affect water-blanketing phenomenon and oil remains shielded throughout the water continuous region. That is why; $f_{0}$ value does not change much w.r.t. WC value in this region.

However, we can estimate the WC value by measuring the dielectric loss of the overall fluid in water continuous region. As the WC value changes, overall loss of the medium increases because the saline water is the major contributor to the dielectric loss. As mentioned earlier that the dielectric loss of the medium can be calculated using resonator's quality (Q) factor. Fig. 14 shows the Q-factor of the sensor in the saline water medium of $44,400 \mathrm{ppm}(4.4 \%)$.

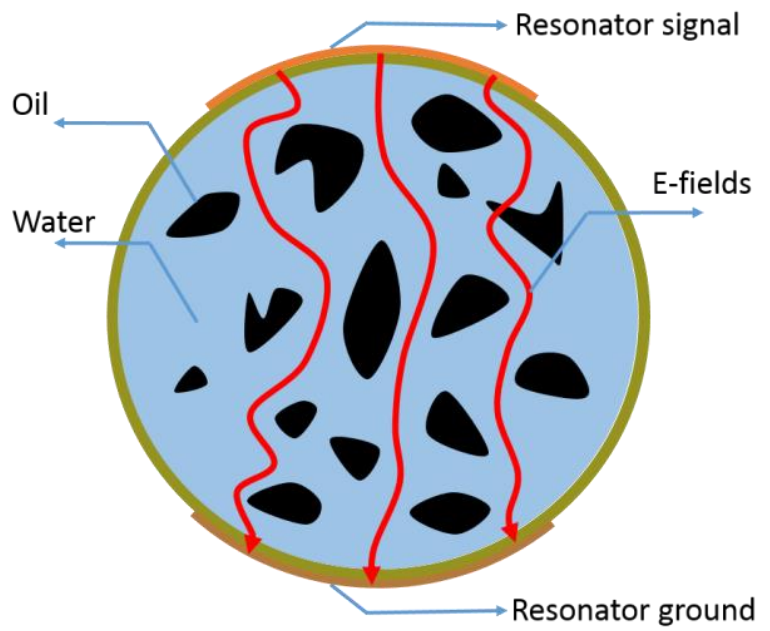

Fig. 13. Dummy demonstration of water blanketing effect in water continuous region 


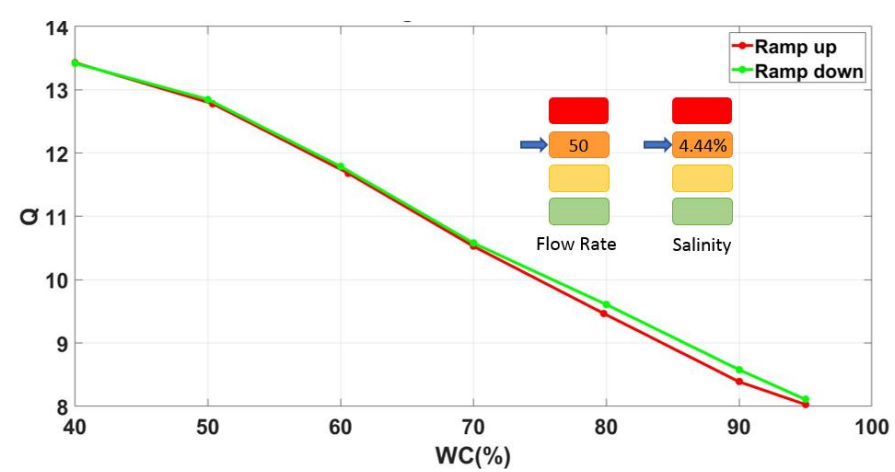

Fig. 14. $Q$ response of the microwave WC sensor in water continuous region

As mentioned earlier that Q-factor has an inverse relationship with the dielectric loss of the fluid. It means that the fluid with high dielectric loss will give lower Q-value and vice versa. High WC value corresponds to higher amount of water in the fluid, which means that overall loss of the fluid is large and we observe low Q at high WC values as is evident from Fig. 14.

It is worth mentioning that the dielectric loss of the fluid is not only the function of the WC but also depends on the salinity of the water as well. That is why; we have characterized our sensor at varying salinity levels ranging from 10,000 $\mathrm{ppm}$ to $85,000 \mathrm{ppm}$ and have plotted the Q-factor versus WC in Fig. 15. The WC sensor works well at even higher salinity levels (up to 200,000 ppm) as evident in Fig. 5 but the sensor could not be tested at extreme salinity conditions due to the limitation of the NORCE flow loop.

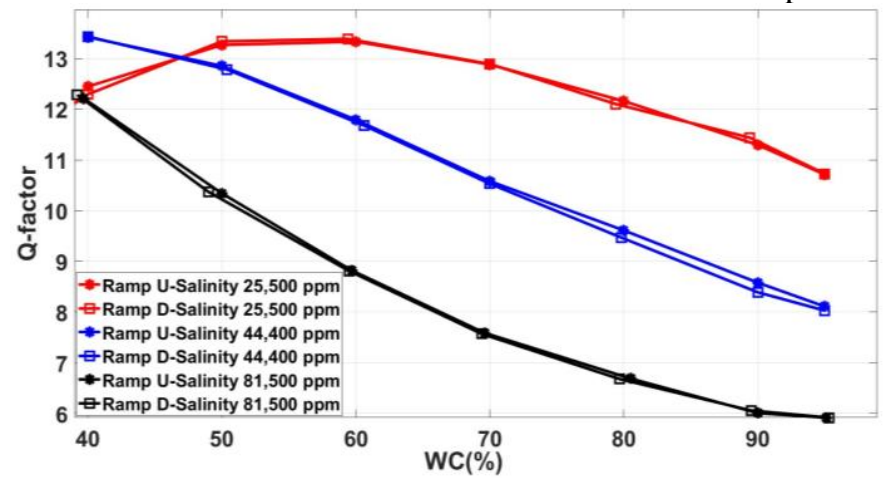

Fig. 15. Water salinity effect on Q-response of the flow meter in water continuous region while ramping up (U) and ramping down (D) the WC value

It can be seen from Fig. 15 that Q-factor curve shifts downwards as the salinity of the medium is increased. The measurements matches well with the theory because dielectric loss increases by increasing the salinity of water which results in lower Q-values. With large data set available for varying salinity levels, we can calibrate out the salinity effect to determine correct WC value in water continuous region.

It can also be observed from Fig. 15 that two set of measurement data has been obtained for each salinity level by ramping up (Ramp U) and ramping down (Ramp D) the WC value. The overlapping values of two set of measurements confirms the repeatability of the sensor.

\section{4) Performance test of the meter}

After characterizing the meter over full WC range in varying salinity levels, we have performed a blind-test to gauge the accuracy of the sensor to measure the WC as well as liquid flow rate. At first, sensor detects the continuous phase using the resonance frequency $\left(f_{0}\right)$. If $f_{0}$ is greater than 150 $\mathrm{MHz}$, oil is considered as the continuous phase and the WC value is determined using $\mathrm{f}_{0}$. On the other hand, 2-phase liquid is considered as water continuous if $\mathrm{f}_{0}$ value is less than 145 $\mathrm{MHz}$ and $\mathrm{WC}$ is determined using Q-factor after calibrating out the effect of salinity. Fig. 16 shows the WC value measured by the proposed sensor, which has been compared against the WC set in the NORCE (previously CMR) flow loop.

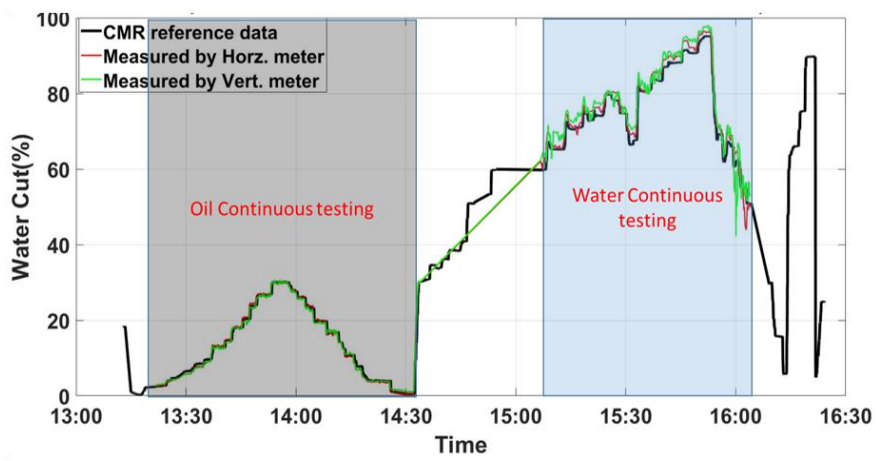

Fig. 16. Comparison of WC value measured by the WC sensors (horizontal and vertical) in relation with the set WC value in the flow loop

Fig. 17 compares the measured liquid flow rate in relation with the set liquid flow rate in the flow loop.

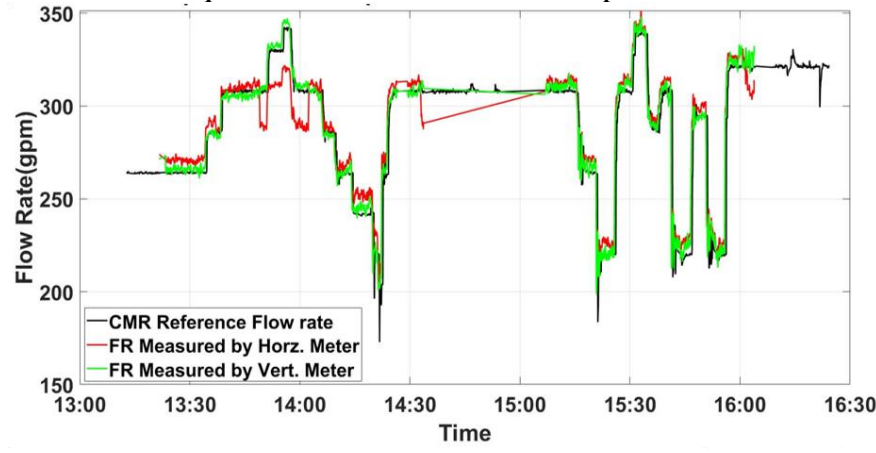

Fig. 17. Comparison of 2-phase liquid flow rate measured by the extended throat venturi (horizontal and vertical) in relation with the set flow rate in the flow loop

Fig. 16 and Fig. 17 show the measured WC and liquid flow rate for two prototypes which were installed horizontally (horz.) and vertically (vert.). Measurement accuracy is the same for both the prototypes, which means that the accuracy of the sensor is not affected by the orientation of the sensor as suggested by simulation results presented in section IIB of this paper.

WC measurement accuracy has been recorded as $\pm 1 \%$ in oil-continuous region and $\pm 2 \%$ in water-continuous region. WC measurement accuracy is slightly less in water-continuous region due to the poor EM shielding of the device and can be improved in future versions of the sensor. Flow rate measurement accuracy in the range of $200 \mathrm{gpm}(6,850 \mathrm{bpd})$ to $350 \mathrm{gpm}(12,000 \mathrm{bpd})$ is around $\pm 3 \%$. The venturi used in the proposed design is capable of measuring the flow rate as low as $1000 \mathrm{bpd}$ but requires more sensitive pressure transducer than used in our current measurement setup. Appropriate transducer can be installed with the venturi based on the intended flow range to measure. 


\section{CONCLUSION}

This paper has presented a novel extended throat venturi based 2-phase flow meter for detection of full range (0-100\%) WC and wide range (1,000-12,000 bpd) of flow rate using a single device. The presented sensor has been designed to withstand harsh field conditions of up to 1000 psi pressure and $125^{\circ} \mathrm{C}$ temperature. The sensor has been demonstrated to achieve accurate WC sensing irrespective of orientation of oil and water inside the pipe. This feature allows this sensor to be installed at any angle without any need of a flow conditioner such as a mixer or blind-tee. Unlike some of the existing meters, presented sensor gives a mean of reliable and nonintrusive WC measurements. Various factors affecting the phase inversion phenomenon have also been investigated in this paper. To compensate the effect of salinity, presented meter has been characterized in variable salinity conditions. In future, this work may be expanded to measure WC value in the presence of gas. At the moment, the sensor assumes that the salinity of produced water is known through some sampling technique. However, in-line salinity measurement will make remote calibration possible and some work needs to done in this regard as well.

\section{REFERENCES}

[1] F.-R. Ahmadun, A. Pendashteh, L. C. Abdullah, D. R. A. Biak, S. S. Madaeni and Z. Z. Abidin, "Review of technologies for oil and gas produced water treatment," Journal of Hazardous Materials, vol. 170, no. 2-3, pp. 530-551, 2009.

[2] B. Bailey, M. Crabtree, J. Tyrie, J. Elphick, F. Kuchuk, C. Romano and L. Roodhart, "Water Control, Oilfield Review," Schlumberger, 2000.

[3] M. Meribout, N. Z. Al-Rawahi, A. M. Al-Naamany, A. Al-Bimani, K. A. Busaidi and A. Meribout, "An Accurate Machine for Real-Time Two-Phase Flowmetering in a Laboratory-Scale Flow Loop," IEEE Transactions on Instrumentation and Measurement, vol. 58, no. 8, pp. 2686 - 2696, 2009

[4] M. Meribout, I. M. Saied and E. A. Hosani, "A New FPGA-Based Terahertz Imaging Device for Multiphase Flow Metering," IEEE Transactions on Terahertz Science and Technology, vol. 8, no. 4, pp. 418 - 426, 2018.

[5] O. H. Unalmis and J. Lievois, "Multiphase flow meter for subsea applications". US Patent 2014/0076547 A1, 20 March 2014.

[6] H. Zhang, L. Zhai, Y. Han, X. Chen, Z. Gao and N. Jin, "Response Characteristics of Coaxial Capacitance Sensor for Horizontal Segregated and Non-Uniform Oil-Water Two-Phase Flows," IEEE Sensors Journal, vol. 17, no. 2, pp. 359 - 368, 2017.

[7] M. A. Karimi, M. Arsalan and A. Shamim, "Design and Dynamic Characterization of an Orientation Insensitive Microwave Water-Cut Sensor," IEEE Transactions on Microwave Theory and Techniques , vol. 66, no. 1, pp. 530 - 539, 2017.

[8] B. Hjertaker, S.-A. Tjugum, E. Hammer and G. Johansen, "Multimodality tomography for multiphase hydrocarbon flow measurements," IEEE Sensors Journal, vol. 5, no. 2, pp. 153 - 160, 2005 .

[9] J. Wei, N. Jin, E. Lian, D. Wang, Y. Han and L. Zhai, "Measurement of Water Holdup in Oil-in-Water Flows Using Three-Channel Conductance Probe With Center Body," IEEE Sensors Journal, vol. 18, no. 7, pp. 2845 - 2852, 2018.

[10] M. Meribout, N. Z. Al-Rawahi, A. M. Al-Naamany, A. Al-Bimani, K. Al-Busaidi and A. Meribout, "A Multisensor Intelligent Device for Real-Time Multiphase Flow Metering in Oil Fields," IEEE Transactions on Instrumentation and Measurement, vol. 59, no. 6, pp. $1507-1519,2010$.
[11] M. Meribout, F. Shehaz, I. M. Saied, Q. A. Bloohsi and A. AlAmri, "High Gas Void Fraction Flow Measurement and Imaging Using a THzBased Device," IEEE Transactions on Terahertz Science and Technology, vol. 9, no. 6, pp. 659 - 668, 2019.

[12] R. Mirzavand, M. M. Honari and P. Mousavi, "High-Resolution Balanced Microwave Material Sensor With Extended Dielectric Range," IEEE Transactions on Industrial Electronics, vol. 64, no. 2, pp. 1552 - 1560, 2017.

[13] R. Mirzavand, M. M. Honari and P. Mousavi, "Direct-Conversion Sensor for Wireless Sensing Networks," IEEE Transactions on Industrial Electronics, vol. 64, no. 12, pp. 9675 - 9682, 2017.

[14] M. H. Zarifi, S. Deif, M. Abdolrazzaghi, B. Chen, D. Ramsawak, M. Amyotte, N. Vahabisani, Z. Hashisho, W. Chen and M. Daneshmand, "A Microwave Ring Resonator Sensor for Early Detection of Breaches in Pipeline Coatings," IEEE Transactions on Industrial Electronics, vol. 65, no. 2, pp. 1626 - 1635, 2018.

[15] Mohammad Abdolrazzaghi, Mohammad Hossein Zarifi, Mojgan Daneshmand, and Cedric F. A. Floquet," Contactless asphaltene solid particle deposition monitoring using active microwave resonators," in 2016 IEEE SENSORS.

[16] H. Zhang, L. Zhai, Y. Han, X. Chen, Z. Gao and N. Jin, "Response Characteristics of Coaxial Capacitance Sensor for Horizontal Segregated and Non-Uniform Oil-Water Two-Phase Flows," IEEE Sensors Journal, vol. 17, no. 2, pp. 359 - 368, 2017.

[17] S. Mohammadi, B. Wiltshire, M. C. Jain, A. V. Nadaraja, A. Clements, K. Golovin, D. J. Roberts, T. Johnson, I. Foulds, M. H. Zarifi, "Gold Coplanar Waveguide Resonator Integrated With a Microfluidic Channel for Aqueous Dielectric Detection" IEEE Sensors Journal, vol. 20, no. 17, pp. 9825-9833, 2020

[18] C. Tan, W. Dai, H. Wu and F. Dong, "A Conductance Ring Coupled Cone Meter for Oil-Water Two-Phase Flow Measurement," IEEE Sensors Journal, vol. 14, no. 4, pp. 1244 - 1252, 2014

[19] M. Zamora and M. P. Henry, "An FPGA Implementation of a Digital Coriolis Mass Flow Metering Drive System," IEEE Transactions on Industrial Electronics, vol. 55, no. 7, pp. 2820 - 2831, 2008

[20] L. Xu, W. Zhou, X. Li and M. Wang, "Wet-Gas Flow Modeling for the Straight Section of Throat-Extended Venturi Meter," IEEE Transactions on Instrumentation and Measurement, vol. 60, no. 6, pp. 2080 - 2087, 2011.

[21] A. Gryzlov, E. Undheim, E. Nyfors, L. Jordaan, S. J. Alvær and E. Steinsland, "Challenges with salinity measurements in multiphase flow metering," in 31st North Sea Flow Measurement Workshop 2013, 2013.

[22] NORCE, "NORCE Multiphase Flow Loop," [Online]. Available: https://www.norceresearch.no/en/norce-flerfaserigg. 